\title{
UNDERLYING CRITERIA IN VALUED PREFERENCE RELATIONS
}

\author{
J. MONTERO, J. YÁÑEZ, D. GÓMEZ \\ Faculty of Mathematics, Complutense University \\ Madrid, Spain \\ E-mail: monty@mat.ucm.es \\ J. GONZÁLEZ-PACHÓN \\ Dept. Artificial Intelligence, Politechnic University of Madrid \\ Boadilla del Monte, Spain \\ E-mail: jgpachon@fi.upm.es \\ V. CUTELLO \\ Dept. Mathematics, University of Catania \\ Catania, Italy \\ E-mail: cutello@dmi.unict.it
}

\begin{abstract}
Translation of Classical Dimension Theory into a valued context should allow a comprehensible view of alternatives, by means of an informative representation, being this representation still manageable by decision makers. In fact, there is an absolute need for this kind of representations, since being able to comprehend a valued preference relation is most of the time the very first difficulty decision makers afford, even when dealing with a small number of alternatives. Moreover, we should be expecting deep computational problems, already present in classical crisp Dimension Theory. A natural approach could be to analyze dimension of every $\alpha$-cut of a given valued preference relation. But due to complexity in dealing with valued preference relations, imposing max-min transitivity to decision makers in order to assure that every $\alpha$-cut defines a crisp partial order set seems quite unrealistic. In this paper we propose an alternative definition of crisp dimension, based upon a general representation result, that may allow the possibility of skipping some of those computational problems.
\end{abstract}

Key words: Valued Relations, Dimension Theory.

\section{Introduction}

Classical Dimension Theory, as introduced by Dushnik and Miller ${ }^{4}$, refers to the representation of partial orders as an intersection of linear orders. From a multicriteria perspective, dimension suggests the minimum number of simple criteria explaining a given transitive preference, being in addition non reflexive and antisymmetric. In this sense, dimension of crisp preferences seems to be a promising tool, hopefully useful for a better understanding of crisp strict binary preference relations, meanwhile they define a partial order set. Three 
main criticism are immediate: (A) quite often preferences are not crisp, but valued; (B) even if they are crisp, too often they do not define a partial order set; (C) even if they define a crisp partial order, it is too difficult to evaluate such a dimension.

In fact, Yannakakis ${ }^{18}$ proved that determining if a partial order has dimension $\mathrm{n}, n \geq 3$, is a NP-complete problem. However, the algorithm proposed by Yáñez and Montero ${ }^{19}$ allows the evaluation of dimension of medium size partial orders. So, something can be done despite criticism (C).

Since valued preferences may contain much more information than crisp preferences, it would be very useful if we can develop manageable representations, perhaps bringing into this context classical dimension concept. Montero et al. ${ }^{12}$ proposed to evaluate dimension for each alpha-cut (see also Van de Walle and Turoff ${ }^{17}$ ). But it is then essential to delete restriction to partial order sets: it does not seem realistic at all to assume that valued preferences defined by decision makers will define always appropriate alpha-cuts (too many values need to be consistently assigned). We do need a general representation theorem for arbitrary crisp strict preferences in order to fully overcome criticism (B), although the deep algorithmic complexity problem may still remain (the underlying representation problem was initially addressed by GonzálezPachón et al. ${ }^{8}$ ).

In this paper we analyze some consequences of the previous results in the concept of dimension function based upon a general representation for $\alpha$-cuts, as an alternative to other attempts to bring dimension into a valued context. We also explore in this paper some alternative approaches in order to partially bypass algorithmic complexity, by means of approximate representations.

We should point out the key relevance of any informative geometrical representation in decision making, meanwhile each representation may give a better insight on the decision problem we are being faced to. In this sense, modern multi criteria procedures are giving an increasing role to representation software (see, e.g., Macharis and Brans ${ }^{10}$ ), becoming knowledge aid tools rather than decision aid tools.

We shall first give a short overview on classical crisp theory in order realize that the standard approach of applying the crisp concept to the sequence of $\alpha$-cut is too restrictive when applied into our context.

\section{Crisp Dimension Theory}

Given a finite set of alternatives $X=\left\{x_{1}, x_{2}, \ldots, x_{n}\right\}$, a crisp partial order set $R \subset X \times X$ is characterized by a mapping

$$
\mu^{R}: X \times X \rightarrow\{0,1\}
$$


being

1. non reflexive: $\mu^{R}\left(x_{i}, x_{i}\right)=0 \quad \forall x_{i} \in X$;

2. antisymmetric: $\mu^{R}\left(x_{i}, x_{j}\right)=1 \Rightarrow \mu^{R}\left(x_{j}, x_{i}\right)=0$; and

3. transitive: $\mu^{R}\left(x_{i}, x_{j}\right)=\mu^{R}\left(x_{j}, x_{k}\right)=1 \Rightarrow \mu^{R}\left(x_{i}, x_{k}\right)=1$

It is therefore assumed that $\mu^{R}\left(x_{i}, x_{j}\right)=1$ means that alternative $x_{i}$ is strictly better than $x_{j}\left(\mu^{R}\left(x_{i}, x_{j}\right)=0\right.$ otherwise $)$.

Dimension of a crisp partial order $R$ was initially defined by Dushnik and Miller ${ }^{4}$ as the minimum number, $\operatorname{dim}(R)$, of linear orders (i.e., complete partial orders) whose intersection is $R$. Being $R$ a partial order set (poset) with dimension $d$, each element $x_{i} \in X$ can be represented in the real space $\left(x_{i}^{1}, \ldots, x_{i}^{d}\right) \in \Re^{d}$ in such a way that

$$
\mu^{R}\left(x_{i}, x_{j}\right)=1 \quad \Longleftrightarrow \quad x_{i}^{k}>x_{j}^{k} \quad \forall k \in\{1, \ldots d\}
$$

for all $x_{i}, x_{j} \in X$ (see also Trotter ${ }^{15}$ ). Such a definition was possible due to Szpilrajn ${ }^{14}$, who had previously proven that every partial order may be represented as an intersection of linear orders, which may be understood as pre-existing underlying criteria in decision maker's mind.

We can also denote $\mu^{R}\left(x_{i}, x_{j}\right)=1$ (i.e., $x_{i} R x_{j}$ or $\left(x_{i}, x_{j}\right) \in R$ ) by $x_{i}>$ $x_{j}$. By $\left[x_{i(1)}, x_{i(2)}, \cdots, x_{i(d)}\right]$ we shall denote here the linear order such that $x_{i(j)}>x_{i(k)}$ for all $j<k$.

\section{Valued preference relations}

In this context, a valued preference relation will assign to each pair of alternatives $\left(x_{i}, x_{j}\right)$ the degree to which first alternative $x_{i}$ is considered strictly better than second alternative $x_{j}$, in such a way that a mapping

$$
\mu: X \times X \rightarrow[0,1]
$$

is being defined (see Zadeh ${ }^{20}$ ).

Of course, some consistency assumptions use to be made within such valued preference relations context. Of course, $\mu\left(x_{i}, x_{i}\right)=0$ seems to be a must if we really deal with strict preferences. And it is often assumed that such a preference relation is max-min transitive, i.e.,

$$
\mu\left(x_{i}, x_{k}\right) \geq \min _{j}\left\{\mu\left(x_{i}, x_{j}\right), \mu\left(x_{j}, x_{k}\right)\right\}
$$

for all $x_{i}, x_{k} \in X$. 
A standard analysis of a given valued preference relation (see GonzálezPachón et al. ${ }^{7}$ ) is to analyze properties of the sequence of its $\alpha$-cuts: for each $\alpha \in(0,1]$, the $\alpha$-cut of a valued preference relation $\mu$ is defined as the crisp binary relation $R_{\mu}^{\alpha}$ in $X$ such that

$$
x_{i} R_{\mu}^{\alpha} x_{j} \Longleftrightarrow \mu\left(x_{i}, x_{j}\right) \geq \alpha
$$

Hence, if we are willing to apply this standard approach to the dimension concept, we should be assuring that every $\alpha$-cut is a partial order set. But this is not true in general, although max-min transitivity implies that every $\alpha$-cut is $\max$-min transitive (see, e.g., Montero et al. ${ }^{12}$ ). For example, even being $\mu$ max-min transitive, if

$$
\alpha_{i j}=\min \left\{\mu\left(x_{i}, x_{j}\right), \mu\left(x_{j}, x_{i}\right)\right\}>0
$$

then we have that $R_{\mu}^{\alpha_{i j}}$ is not antisymmetric.

Moreover, imposing max-min transitivity or any other analogous consistency condition seems to be too restrictive in practice, due to the extreme difficulties decision maker will find in assigning all those $n(n-1)$ degrees of preference, as it can be easily checked in the literature (quite often we search for a max-min transitive valued binary relation being in some sense close to data; see, e.g., Montero and Tejada ${ }^{11}$ ).

\section{General representation of a crisp partial order}

The following result shows that any crisp strict preference relation can be represented in terms of unions of intersections of linear orders, no matter if incomparability or intransitivity or even cycles are present (see González-Pachón et al. ${ }^{7}$ ).

Theorem.- Let $X=\left\{x_{1}, \ldots, x_{n}\right\}$ be a finite set of alternatives, and let us consider

$$
\mathcal{C}=\{L / L \text { linear order on } X\}
$$

Then for every non-reflexive crisp binary relation $R$ on $X$ there exist a family of linear orders $\left\{L_{s t}\right\}_{s, t} \subset \mathcal{C}$ such that

$$
R=\bigcup_{s} \bigcap_{t} L_{s t}
$$

Hence, the generalized dimension, $\operatorname{Dim}(R)$, of a crisp strict preference binary relation $R$ can be defined as the minimum number of different linear orders, $L_{s t}$, such that

$$
R=\bigcup_{s} \bigcap_{t} L_{s t}
$$


For example, if we have two alternatives, $X=\left\{x_{1}, x_{2}\right\}$, then incomparability $R_{1}$ between both alternatives

$$
\mu^{R_{1}}\left(x_{1}, x_{2}\right)=\mu^{R_{1}}\left(x_{2}, x_{1}\right)=0
$$

can be represented as

$$
R_{1}=L_{1} \cap L_{2}
$$

where $L_{1}=\left[x_{1}, x_{2}\right]$ and $L_{2}=\left[x_{2}, x_{1}\right]$. Analogously, a cycle $R_{2}$ between both alternatives

$$
\mu^{R_{2}}\left(x_{1}, x_{2}\right)=\mu^{R_{2}}\left(x_{2}, x_{1}\right)=1
$$

can be represented as

$$
R_{2}=L_{1} \cup L_{2}
$$

It is important to notice that our generalized dimension does not extends classical dimension. Although quite often partial order sets have the same dimension in both senses, the following result shows a partial order set such that its generalized dimension is not equal to its classical dimension (see GonzálezPachón et al. $\left.{ }^{6}\right)$.

Example.- Let $X=\left\{y_{1}, \cdots, y_{n}, z_{1}, \cdots, z_{n}\right\}$ a finite set of $2 n$ alternatives, $n \geq 6$, and let us consider the following partial order set $\hat{R}$ :

- $\mu^{\hat{R}}\left(y_{i}, z_{j}\right)=1$ if $i \neq j$, and

- $\mu^{\hat{R}}\left(x, x^{\prime}\right)=0$ otherwise.

Then $\operatorname{Dim}(\hat{R})<\operatorname{dim}(\hat{R})$.

\section{Generalized dimension function}

Once we have fully generalized and therefore overcome main restriction of classical dimension theory, the above general representation result for crisp strict relations can be translated to a valued context in order to relax the normative approach given in Montero et al. ${ }^{12}$ and evaluate the generalized dimension $\operatorname{Dim}\left(R_{\mu}^{\alpha}\right)$ for each $\alpha$-cut, $\alpha \in(0,1]$.

Hence, we no longer need to impose that every $\alpha$-cut defines a partial order set, although it must be pointed out again that such a generalized dimension is not an extension of classical dimension. This result may suggest that classical dimension should be revised in order to check the intuitive significance of both approaches.

Moreover, if we take into account that rationality is not an univoque concept (see Cutello and Montero ${ }^{2}$ ), we may think that linear orders may be not 
the unique absolute rational crisp preference relations. It may be the case then that we can develop a consistency concept subject to our particular available computational abilities (of a linguistic perhaps, see Herrera et al. ${ }^{9}$ ), in such a way that the above pointed out deep computational problems can be in some way bypassed (see González-Pachón et al. ${ }^{6}$ ). The following result, for example, suggests that most families of binary relations based upon a unique basic structure up to permutation of all alternatives can be taken into account in order to get similar representations.

Theorem.- Let $\mathcal{C}$ be a non-empty family of crisp strict binary preference relations defined on a finite set of alternatives $X$ such that

$$
\cap\{R \mid(a, b) \in R\}=\{(a, b)\} \quad \forall(a, b) \in X \times X
$$

Then any strict binary preference relation can be represented as the union of intersections of elements in $\mathcal{C}$.

\section{Final comments}

The key issue for future research is how to exploit information from the generalized dimension function of a valued preference relation $\mu$, defined as the mapping

$$
\left\{D(\alpha)=\operatorname{Dim}\left(R_{\mu}^{\alpha}\right)\right\}_{\alpha \in(0,1]}
$$

Such a research should be developed considering different alternative rationalities, and taking into account that such a (generalized) dimension may not be giving a comprehensive understanding of a given crisp preference unless some informative (generalized) representation is associated. Some experiences are actually being run in order to learn from the information contained in those representations.

Some alternative approaches to the dimension concept of valued preference relations can be found in the literature, taking a quite different point of view. Adnadjevic ${ }^{1}$, for example, has proposed an alternative definition of dimension for valued preference relations based upon the notion of multichain, but assuming strong consistency properties on the decision maker preference relation. On the contrary, Ovchinnikov ${ }^{13}$ propose a different dimension concept in terms of an underlying representation which appears to be too difficult to be managed by decision makers. Analogous criticism applies to Fodor and Roubens ${ }^{5}$ and Doignon and Mitas ${ }^{3}$ (both based upon a previous result of Valverde ${ }^{16}$, they represent valued preferences by means of another canonical valued preferences).

Crisp dimension approach applied to all $\alpha$-cuts of a valued preference relation, as proposed in González-Pachón et al. ${ }^{7}$, seems an interesting hint for 
decision makers in practice, and they are in fact taken into account in Doignon and Mitas ${ }^{3}$ in order to obtain operative bounds.

\section{Acknowledgments}

This research has been partially supported by the Government of Spain, grant PB98-0825.

\section{References}

1. D. Adnadjevic: Dimension of fuzzy ordered sets. Fuzzy Sets and Systems 67:349-357 (1994).

2. V. Cutello and J. Montero: Fuzzy rationality measures. Fuzzy Sets and Systems 62:39-54 (1994).

3. J.P. Doignon and J. Mitas: Dimension of valued relations. European Journal of Operational Research 125:571-587 (2000).

4. B. Dushnik and E.W. Miller: Partially ordered sets. American Journal of Mathematics 63:600-610 (1941).

5. J.C. Fodor and M. Roubens: Structure of valued binary relations. Mathematical Social Sciences 30:71-94 (1995).

6. J. González-Pachón, D. Gómez, J. Montero, J. Yáñez: Searching for the dimension of valued preference relations. Submited.

7. J. González-Pachón, D. Gómez, J. Montero, J. Yáñez: Soft dimension theory. Fuzzy Sets and Systems, to appear.

8. J. González-Pachón and S. Ríos-Insua: Mixture of maximal quasi orders: a new approach to preference modelling. Theory and Decisions 47:73-88 (1999).

9. F. Herrera, E. Herrera-Viedma and L. Martínez: A hierarchical ordinal model for managing unbalanced linguistic terms sets based on the linguistic 2-tuple model. Proceedings EUROFUSE, Workshop of Preference Modelling and Applications (Granada, April 25-27, 2001), 201-206.

10. C. Macharis and J.P. Brans: The GDSS Promethee Procedure. Journal of Decision Systems 7:283-307 (1998).

11. J. Montero and J. Tejada: Some problems on the definition of fuzzy preference relations. Fuzzy Sets and Systems 20:45-53 (1986).

12. J. Montero, J. Yáñez and V. Cutello: On the dimension of fuzzy preference relations. Proceedings International ICSC Symposium on Engineering of Intelligent Systems, University of La Laguna (1998), vol. 3, $38-33$.

13. S.V. Ovchinnikov: Representation of transitive fuzzy relations. In: H.J. Skala, S. Termini and E. Trillas (eds.). Aspects of Vagueness (Reidel, 
Amsterdam, 1984), 105-118.

14. E. Szpilrajn: Sur l'extension de l'ordre partiel. Fundamenta Mathematicae 16:386-389 (1930).

15. W.T. Trotter: Combinatorics and Partially Ordered Sets. Dimension Theory (The Johns Hopkins University Press, Baltimore and London, 1992).

16. L. Valverde: On the structure of F-indistinguishability operators. Fuzzy Sets and Systems 17:313-328 (1985).

17. B. van de Walle and M. Turoff: Towards group agreement: the significance of preference analysis. Proceedings EUROFUSE, Workshop of Preference Modelling and Applications (Granada, April 25-27, 2001), 85-92

18. M. Yannakakis: On the complexity of the partial order dimension problem. SIAM Journal of Algebra and Discrete Mathematics 3:351-358 (1982).

19. J. Yáñez and J. Montero: A poset dimension algorithm. Journal of Algorithms 30:185-208 (2000).

20. L.A. Zadeh: Similarity relations and fuzzy orderings. Informations Sciences 3:177-200 (1971). 